\title{
El desarrollo de la personalidad creativa en el estudiante de la Licenciatura en Intervención Educativa
}

\section{The development of the creative personality in the student of the Degree in Educational Intervention}

Hermelinda Bañuelas Bustillos ${ }^{1}$

\begin{abstract}
Resumen
El problema que se aborda en este trabajo es ¿Cómo desarrollar el pensamiento creativo en los estudiantes de sexto semestre de la Licenciatura en Intervención Educativa de la Universidad Pedagógica Nacional del Estado de Chihuahua Campus Camargo? los objetivos y las preguntas de indagación inquieren sobre: la historia personal y familiar de los estudiantes, los aspectos que determinan el desarrollo de la personalidad creativa, la posibilidad de su potenciación mediante estrategias específicas, el fundamento que se debe poseer, así como el ambiente que favorece el pensamiento creativo. El proyecto tiene actividades cognoscitivas, plásticas, lingüísticas y físicas para favorecer la imaginación, el ingenio, la inventiva, la generación de ideas y la fluidez, así como la flexibilidad de pensamiento. Los resultados revelan que los estudiantes, a pesar de que se mostraron abiertos y dispuestos al trabajo y tuvieron oportunidad de revisar detenidamente el conocimiento mínimo para entender el tema, no realizaron las actividades como se pretendía porque existía un clima grupal poco favorable y el tiempo dedicado no fue suficiente, por tanto, las sugerencias van en el sentido de otorgar más tiempo extendiendo los trabajo a un año y dar más tiempo a la duración de las actividades para lograr mejores resultados.
\end{abstract}

\section{Palabras clave}

Creatividad, desarrollo, pensamiento, personalidad.

\footnotetext{
${ }^{1}$ Hermelinda Bañuelas Bustillos. Profesora de la Universidad Pedagógica Nacional del Estado de Chihuahua, Campus Camargo, México. Correo electrónico: lidesi070301@yahoo.com.mx
}

ID: http://orcid.org/0000-0002-7555-7327 
RECIE. Revista Electrónica Científica de Investigación Educativa Vol. 4, núm. 2, enero-diciembre 2019, pp. 1337-1348.

\begin{abstract}
The problem addressed in this work is How to develop creative thinking in the sixth semester students of the Universidad Pedagógica Nacional del Estado de Chihuahua, Campus Camargo? The objectives and the questions inquire about: the personal and family history of the students, the aspects that determine the development of the creative personality, the possibility of their empowerment through specific strategies, the foundation that must be possessed, as well as the environment that favors creative thinking. The project has cognitive, plastic, linguistic and physical activities to favor the imagination, the ingenuity, the inventiveness, the generation of ideas and fluency, as well as the flexibility of thought. The results reveal that the students, although they were open and willing to work and had the opportunity to carefully review the minimum knowledge to understand the topic, did not carry out the activities as intended because there was an unfavorable group climate and the time dedicated it was not enough, therefore, the suggestions go in the sense of granting more time extending the work to a year and giving more time to the duration of the activities to achieve better result.
\end{abstract}

\title{
Keywords
}

Creativity, development, thought, personality.

\section{Introducción}

Las habilidades de pensamiento crítico y creativo deberían considerarse como un punto central del currículum para fomentarse en el contexto de la enseñanza escolar, pues está demostrado en distintos estudios sobre el tema, que mediante actividades que impliquen retos cognitivos, plásticos, físicos, matemáticos, lingüísticos, de inventiva e imaginación, trabajarlas propicia mayor conexión neuronal que hace más inteligentes a las personas y se desarrollan habilidades para resolver problemas cotidianos, hay varios estudios que aportan experiencias en educación superior, unas favorables y otras, no tanto, por lo que con este trabajo se intenta dar cuenta de una experiencia más, obtenida luego de aplicar un programa que incluye actividades cognitivas, plásticas, lingüísticas y físicas.

\section{Estado del arte}

Los trabajos del estado del arte en esta investigación son: 'La gimnasia cerebral como estrategia para desarrollo de la creatividad en los estudiantes' de Romero, Cueva y Barbosa (2014); 'Efectos de un programa 
para desarrollar las habilidades creativas' de Larraz y Torres (2012); y 'Descubriendo la creatividad en estudiantes universitarios: preferencias y tendencias mediante la prueba DTC' de la Universidad Nacional Autónoma de México y la Universidad de Barcelona, España de Esquivias y De la Torre (2010).

\section{Planteamiento del problema}

- ¿Qué actividades favorecen el desarrollo del pensamiento creativo en los estudiantes del sexto semestre de la Licenciatura en Intervención Educativa de la UPENECH Campus Camargo?

- Preguntas de investigación

- ¿Cuáles son las experiencias personales, familiares y sociales que han determinado el desarrollo del pensamiento creativo en los estudiantes de sexto semestre de la Licenciatura en Intervención Educativa?

- ¿Cómo incidir en la mejora de los aspectos cognitivos, afectivos y volitivos de la personalidad creativa en los estudiantes del grupo?

- ¿Qué condiciones se requieren para desarrollar y mejorar la creatividad de los estudiantes?

\section{Objetivos}

- Conocer las experiencias personales y familiares que determinado el pensamiento creativo de los estudiantes del grupo de sexto semestre de la Licenciatura en Intervención Educativa.

- Implementar actividades para potenciar el pensamiento creativo en los estudiantes del grupo estudiado a fin de mejorar su capacidad creadora.

- Crear las condiciones necesarias para el desarrollo del pensamiento creativo en los estudiantes.

- Desarrollar los rasgos de la personalidad creativa en los estudiantes del grupo.

\section{Referentes teórico-conceptuales}

Los referentes teóricos se encuentran en las aportaciones de Ludivina Sánchez (2009) y Lorenc Guilera (2011), la primera cita a neurólogos como Hans Berger (1929), James Papez, Paul Mc Lean y a estudiosos del tema como: Guilford (1951), De Bono (1970), Perkins (1985), Carlos de León (1999), Paredes (2000), Campirán (2001), Penagos, J.C. \& Aluni (2000), entre otros. La creatividad refiere a la identificación, planteamiento y 
solución de problemas; implica trabajar de forma precisa, constante e intensa, y considera alternativas nuevas. Es sinónimo de innovación, imaginación, originalidad, invención, visualización, intuición y descubrimiento; significa audacia para tomar nuevos caminos, re-crearse constantemente, administrar la vida propia, ser productivo, competitivo y autorrealizarse; significa planear, ajustar la vida de común acuerdo; producir el ambiente donde emerjan las individualidades y que cada quien logre la satisfacción de sus necesidades; significa crear los medios y el entorno propicio para el desarrollo del capital humano. Siguiendo a Rodríguez, M. (1990), se consideran los rasgos de la personalidad creativa, divididos en tres áreas: cognoscitiva, afectiva y volitiva.

\section{Metodología y procedimientos}

El trabajo se realiza siguiendo el paradigma cualitativo, el método es la investigación-acción; las técnicas seguidas son la observación participante y la entrevista; los instrumentos utilizados fueron los guiones de observación y de entrevista. La recuperación de la información se hizo en 20 registros durante un semestre anotando en ellos aspectos como: realización de actividades, interés, disposición, actitudes frente a la actividad, al conocimiento y al grupo, solución de problemas, desempeño escolar y personal; características de las producciones (realización de ejercicios, número de ideas o conceptos generados, fluidez, tiempo de realización, dedicación, explicaciones ofrecidas, comentarios y expresiones manifestadas, novedad en los productos elaborados como colores, figuras etc.) El proceso de sistematización se realizó según las recomendaciones del paradigma de investigación reduciendo la información a datos, agrupándolos en categorías y patrones recurrentes que se integraron en un esquema para proceder al análisis e interpretación de la misma a la luz de la teoría.

El programa de actividades (ver cuadro 1)se trabajó durante un semestre, dedicándole un espacio de 15 a 30 minutos en las sesiones de clase de la materia: El desarrollo de habilidades de pensamiento en el periodo enero-junio de 2016; está conformado por diez ejercicios y actividades que retan a moverse distinto y por espacios diferentes a los conocidos (seguir rutas diferentes para ir a la escuela y al trabajo), recordar objetos existentes en determinados lugares, vestirse de manera diferente (combinando prendas), caminar para atrás y ejercitarse físicamente como se recomienda desde el pensamiento lateral; de manera simultánea, se plantearon diez ejercicios escritos, como inventar cuentos, adivinanzas y 
palabras asociadas, acertijos y sudokus encontrados en páginas de internet especializadas y en obras sobre el tema.

Se trabajaron en cada sesión de clases un ejercicio físico externo y un ejercicio cognitivo, plástico o lingüístico en el salón de clase sumando un total de 40 ejercicios; se evaluó con ítems como: experiencia, visión de las cosas, respuestas diversas, percepción de los demás, consciencia de cambios en la forma de razonar etc.

El programa de actividades se integra en fichas para que estén a la mano y sea más fácil el trabajo, se agrupan en lingüísticas, plásticas, físicas y mentales para potenciar la fluidez, flexibilidad, originalidad y elaboración de ideas.

\section{Resultados y discusión}

El diagnóstico realizado revela que pocos estudiantes han tenido experiencias creativas importantes en su contexto familiar, reconocen la trascendencia en el éxito personal y los estudios: - Mi mamá me contaba y me leía cuentos por las tardes y decía ahora tú cuéntame un cuento a mí. Me gustaba imaginar lo que me leían, se me hacía divertido, tres estudiantes refieren actividades realizadas en trabajos recientes. -Trabajé en una guardería, me ponían a hacer dibujos para decorar pasillos y salas. -Trabajé en una papelería envolviendo regalos de manera llamativa y fuera de lo común, hacíamos manualidades y cosas bonitas.

Algunos hacen referencia a las experiencias recientes sobre el desarrollo de la creatividad, en ellas dicen, han podido poner en juego su capacidad creadora ya que han tenido que generar ideas, pensar, procurar materiales, interactuar con ellos, diseñar y elaborar estructuras y formas; resaltan su experiencia en las bellas artes baile, actuación, diseño de escenarios diversos -Hemos arreglado carros alegóricos y escenografías de programas. Somos buenos para decorar y hacer cosas bonitas y baratas (DC: 26/05/16). Asesoras: - ¡Son muy creativos! (DC: 4/feb/16). Para el carro tuvimos que pensar y generar ideas ¡Ni te imaginas! ¡Rápido se armó el concepto! (EM2: 2/mar/16).

La información revela discordancias: para una parte del grupo, la experiencia es favorable - Una alumna platica su experiencia mirando y sonriendo a sus compañeros que la definen como creativa (DC: 16/feb/16); para otros no, no comentan ni argumentan, no muestran entusiasmo ni ganas de realizar las actividades y ante la pregunta contestan vagamente yo no me acuerdo de haber hecho algo así (DC: 16/feb/16).

Para algunas asesoras el grupo está bien, pero les falta actitud creativa, -Son así porque en una buena parte de los cursos que han llevado 
las maestras los ponían a exponer, hacen materiales bonitos, pero les falta reflexión (EM2: 2/mar/16).

Los resultados de la aplicación de las actividades revelan que al inicio del programa se presentan conflictos grupales entre estudiantes y la tutora responsable del proyecto y frecuentemente la directora tiene que mediar, se dialoga y acuerdan cosas, Yo así no puedo trabajar, plantéenlo con la directora y nos vemos hasta que ustedes digan otra cosa... Directora: están dispuestos a seguir, piden cambios en la metodología (DC: 4/may/16). Al cierre del curso las cosas mejoran, sorprenden estudiantes poco participativos -Una alumna se levanta y apoya a la compañera que está en el pizarrón intentando una explicación, ambas se muestran entusiastas y participan (DC: 17/may/16).

Con las actividades físicas se logra que los estudiantes reconozcan la importancia del ejercicio físico para favorecer el desarrollo de la creatividad, pero se presentan resistencias en la realización de las actividades: un poco por flojera, por rutina y porque había tensión y rechazo a aceptar el trabajo de la asesora responsable del proyecto: - ¡Mmmm se me olvidó! - ¡No se puede, sólo hay un camino! - ¡No pude porque me levanté tarde y todo lo hice muy rápido! - ¡Yo sólo rodeé el mercado! - ¿Nadie más hizo el ejercicio? Niegan moviendo la cabeza - ¡Se siente extraño! ¡Es difícil ¡Lo intenté, pero me desesperé! - ¡Se me olvidó!

Los ejercicios trabajados en clase resultan más interesantes y se batalla menos para que las lleven a cabo, se puede afirmar que estas hasta les emocionan pues se escuchan exclamaciones como: -¡Hey! ¡Qué no vamos a bailar! ¡Hay que bailar! - ¡Hay que estimular al cerebro maestra! (DC: 9/mar16), esto permite afirmar que el hecho de que algunos pertenecen a los equipos de tochito, vólibol y básquetbol o a grupos de baile, donde han desarrollado bastante corporeidad o consciencia de su cuerpo caracterizándoles el interés por las clases, la disposición para realizar las tareas y actividades escolares y una buena participación y desempeño. Muchos de ustedes ya practican algún deporte o arte, han ejercitado bastante su cerebro, pero nunca está de más, así se puede ser más inteligente (7/feb/16). -Has un esfuerzo - ¡Sí! (DC: 16/mar).

En la generación y representación de ideas los estudiantes realizan las actividades y retos que se indicaban no para abstraer y resumir ideas de otros, sino para generarlas, en "las constelaciones" (media cartulina negra con puntos blancos), se encuentran tres subcategorías:

Primera, once presentan de diez a veinte figuras con color; ellos no batallan para estudiar y trabajar en clase, obtienen buenas notas, toman 
apuntes, cumplen con tareas, la actitud es buena, muestran gusto, disfrute y entusiasmo - ¡Listo, ya está mi dibujo! - ¡Ya terminé! Mira el dibujo sonriendo ¿Qué te parece? (DC: 14/mar/16).

Segunda, seis constelaciones, figuras con menor sentido, de cinco a diez y usan un sólo color, se quejan al realizar el ejercicio -Esta cartulina no tenía tantos puntos iya me dolió la cabeza! -Ya terminé (melancolía) (DC: 14/mar/16). Los estudiantes cuyas producciones quedan en esta categoría son medianamente dedicados y alcanzan calificaciones de ocho. -Antes yo era muy lista, sacaba diez, no sé qué me pasa (DC: $7 / \mathrm{feb} / 16$ ).

Tercera, once constelaciones, pocas figuras, poco sentido; llama la atención una figura humana a medias con una frase del principito, su autora movilizó todas las habilidades de pensamiento, usó sus experiencias y conocimientos previos, realizó inferencias, estableció relaciones, asoció ideas, es una estudiante regular, tiene capacidad, pero se resiste... al tiempo modifica su actitud. -Se pone de pie, señala palabras explica porque debería ir allí, ha estado muy participativa al igual que... ambas se mostraban poco participativas antes, hoy me han dado la sorpresa en el debate grupal (DC: 11/abr/16).

Otras de las producciones fue completar dibujos que sólo contenía un elemento y había que imaginar un escenario y dibujarlo, los resultados se mueven, se incrementa levemente, antes eran seis los productos que se agrupaban en ella, se mueven de dos a tres en cada categoría. En el ejercicio con líneas las producciones también se mejoran.

En los ejercicios lingüísticos reto de escribir parecía interesante ya que consistía en elaborar pequeños textos que incluían cuentos, poemas, adivinanzas, definiciones, generación de palabras, etc., su elaboración partía de alguna base específica, por ejemplo: las letras del abecedario, una imagen de la lotería, palabras base, objetos o dibujos, la consigna es en el menor tiempo posible.

Los alumnos procesan información y se esfuerzan, apoyan a los menos creativos: ¿Qué quieres poner? -¡No así no va! ¡Quita la i! ¡Va con minúscula! Los ejercicios exigen congruencia con la idea del otro; entre un cuento y otro, aparecen más ideas con mejor lógica el segundo, tuvo el ingrediente de que se pidió realizar en individual, después de un ejercicio físico y mental de elaboración de animales con frutas y plastilina, que requería atención, cortes finos, relacionar conocimientos, ideas y experiencias, lo que facilitó, Hacia los tres meses de la aplicación. El interés estuvo elevado en las frases y conceptos chuscos, en el cuento y las adivinanzas, estas provocaban risa a los estudiantes lo cual es comprensible como señalan Rodríguez y Marhyark (1992) en Guilera (2011) "la 
creatividad genera alegría (...), la gente se comporta agradable, disfruta y usan su inventiva.

Los ejercicios mentales consistieron en completar figuras, llenar círculos con números que daban determinada suma, sumas desglosadas que sugerían diversos resultados pero que tenían una sola respuesta correcta, o sumas cuya resolución requería rapidez, aunque se equivocaran en el resultado. El interés se mide con la escala de la personalidad creadora: 16 contestan que les gustaron algo, cinco que mucho y seis bastante, el resultado es positivo como dice Guilera (2011): El mundo está lleno de personas con aptitudes creativas que no la utilizan porque falta la actitud necesaria.

Se elevó el nivel de complejidad a los retos, en series de figuras que tenía lógica y requirió tiempo -cinco estudiantes se mostraron desesperados: - ¡huy que feo está esto! - ¡No lo encuentro! - ¡ya me cansé! Pero en los ejercicios matemáticos y de memoria estuvieron entusiasmados: analizaban, concentrados ponían en juego sus habilidades de pensamiento, iban y veían de una figura a otra comparando, relacionando, encontrando semejanzas; motivados, reían, socializando y reflexionando: ¡Válgame cómo no lo vi cuando pasó! ¡Por qué yo no recuerdo eso! ¡Ajá! ¡yo si me la supe! ¡Si recordé!

En la mejora de la atmosfera se planearon actividades individuales y grupales con el fin de lograr el ambiente adecuado para realizar las producciones, cuidando la novedad, el valor social; se intentó poner en juego los componentes del potencial humano: creativo, cognoscitivo, afectivo, intelectual y volitivo para generar actitudes necesarias, "un maestro que no es creador no puede enseñar a sus alumnos a ser creadores" (Martínez 1960:6).

Al inicio, algunos se mostraban poco participativos, no lograban calificaciones altas, con ellos se tuvo especial cuidado, mostrando de manera permanente una actitud creativa, resaltando las posibilidades del éxito en el desarrollo de competencias y el mejoramiento del desempeño si se lo proponían. Ayudaron los test tanto de inteligencias múltiples como las de evaluación multifactorial de la creatividad (EMUC) de Sánchez (2006) y Escala de Personalidad Creadora (EPC) de Garaigordobil (2004) para verse e intentar mejorar.

Al cabo de un tiempo los estudiantes repetían las frases que la docente utilizaba, cuando algo salía bien o cuando había un reto o problema que resolver: ¡Bravo! ¡Bien! ¡Aplausos! ¡Estrellita! para otorgar reconocimiento al trabajo o la participación; y si en algún momento no lo 
hacía, reclamaban: no dijo: ¡Bravo! ¡Aplausos! ¿Qué pasó? - ¡Vamos! ¡Pensamiento lateral! (DC: 14/abr/16).

Las consignas cumplieron su objetivo: plantear salidas, los estudiantes hicieron esfuerzos por salir adelante en algunas actividades, retos y dificultades grupales. Al completar un mapa mental les cuesta, pero ante la insistencia comienzan a leer y a estructurar las frases. ¿Qué idea falta? ¿Ya está todo en el mapa? - Puedo escribir que la persona pone en juego sus habilidades creativas cuando resuelve problemas - ¡Sí! es una buena idea. Según Guilera (2011: 169), hacer preguntas sobre la realidad es una de las maneras de generar o motivar el desarrollo del pensamiento creativo".

En el aspecto académico mejoran los alumnos con dificultades, pues el alumno debe saber que de él se espera creatividad para que esté consciente de su propio aprendizaje y se esfuerce en mejorarlo (Landou1957: 112 en Guilera, 2011: 32)".

\section{Conclusiones}

Al concluir el programa los estudiantes son capaces de distinguir las características tanto de la personalidad creativa como de los procesos y los productos creativos, gracias a la revisión de los referentes teóricos; ahora reconocen que hay ejercicios que favorecen el hecho de ser más inteligentes si se trabaja para mejorar la creatividad; una buena parte de ellos, se enfocó y mejoró, aunque sea un poco, sus procesos creativos, modificó conductas y comportamientos favoreciendo la personalidad creativa.

A pesar de que los integrantes del grupo estudiado no realizaron las actividades físicas con tanto entusiasmo, dedicación y empeño al inicio de la aplicación, el trabajo logrado con los ejercicios que se realizaban en salón de clase tiene su mérito. Las producciones creativas que presentaban poca elaboración de ideas al principio, mejoran en la segunda; sin embargo, hay que seguir trabajando.

Si un programa de creatividad incluye actividades y materiales diversos y, además se le destina un mayor tiempo, pueden lograrse mejores resultados. Las actividades deben retar al pensamiento a generar ideas, relacionar palabras y conceptos, inventar cuentos o historias, resolver retos matemáticos, realizar ejercicios de retención y/o memorización y usar la contra-lógica así como realizar actividades físicas y plásticas. 
RECIE. Revista Electrónica Científica de Investigación Educativa

Vol. 4, núm. 2, enero-diciembre 2019, pp. 1337-1348.

Cuadro 1 Plan de intervención.

Cuadro que agrupa las actividades implementadas para desarrollar el pensamiento creativo en los estudiantes del sexto semestre de la Licenciatura en Intervención

Educativa.

\begin{tabular}{|c|c|c|c|}
\hline Actividades & Material & Tiempo & Evaluación \\
\hline $\begin{array}{l}\text { LINGÚSTICAS } \\
\text { Objeto mágico. } \\
\text { Tierra, mar y } \\
\text { aire. } \\
\text { Conexión de } \\
\text { palabras. } \\
\text { El telegrama. } \\
\text { El acordeón. } \\
\text { ABC. } \\
\text { Adivinanzas } \\
\text { personales. } \\
\text { Cuento lotería. } \\
\text { Bolso mágico. } \\
\text { Mi concepto }\end{array}$ & $\begin{array}{l}\text { Cualquier objeto. } \\
\text { Papel. } \\
\text { Lápiz. }\end{array}$ & $\begin{array}{l}15 \text { a } 20 \\
\text { minutos al } \\
\text { inicio de la } \\
\text { sesión de } \\
\text { clase. }\end{array}$ & \\
\hline $\begin{array}{l}\text { FÍSICAS } \\
\text { Caminando hacia } \\
\text { atrás. } \\
\text { Recorridos } \\
\text { alternativos. } \\
\text { Siguiendo rutas } \\
\text { diferentes. } \\
\text { El mural rasgado. } \\
\text { Pequeños bailes. } \\
\text { Manos } \\
\text { tramposas. } \\
\text { Manos } \\
\text { contrarias. } \\
\text { Estimulando el } \\
\text { cerebro. } \\
\text { Chuchuguagua. }\end{array}$ & $\begin{array}{l}\text { Música. } \\
\text { Reproductor. } \\
\text { Papel. } \\
\text { Pegamento. }\end{array}$ & $\begin{array}{l}\text { Lo que ocupa } \\
\text { su traslado a } \\
\text { la } \\
\text { universidad. } \\
\text { Los ejercicios } \\
\text { en clase } \\
10 \text { a } 15 \\
\text { minutos }\end{array}$ & $\begin{array}{l}\text { Flexibilidad } \\
\text { Originalidad } \\
\text { Inventiva } \\
\text { Rapidez }\end{array}$ \\
\hline
\end{tabular}

El desarrollo de la personalidad creativa en el estudiante de la Licenciatura... 


\begin{tabular}{|l|l|l|l|}
\hline MENTALES & $\begin{array}{l}\text { Hojas con líneas. } \\
\text { Sudokus. } \\
\text { Líneas }\end{array}$ & $\begin{array}{l}10 \text { a } 15 \\
\text { minutos }\end{array}$ & \\
imaginarias. & Acertijos. & & \\
letras. & & \\
Relaciones & & \\
forzosas. & & \\
Las desventajas. & & \\
Las alternativas. & & \\
La combinación. & & \\
Interpretamos. & & \\
Acertijos. & & \\
Sudokus. & & \\
\hline PLÁSTICAS & Bolsas de papel. & 20 a 30 & \\
minutos & \\
Títeres. & Papel. & Cada sesion & \\
Deco-botes. & Clips. & de clase & \\
Brainstorming. & Maderitas. & \\
Pintura mágica. & Palillos. & \\
Pintura de sellos. & Tornillos. & \\
Mi personaje & Clavos. & \\
favorito. & Martillo. & \\
Cre-in. & Botes. & \\
Los inventos & & \\
& & \\
\hline
\end{tabular}

\section{Referencias}

DE BONO, Edward. (1998). Pensar Bien. Editorial SELECTOR. Décimo tercera reimpresión, México. 143 pp.

DE SÁNCHEZ, Margarita. (2005). Desarrollo de habilidades de pensamiento. 23ra. Reimpresión. Editorial Trillas. México. 209 pp.

ESQUIVIAS, Serrano María Teresa \& Saturnino, De la Torre De la Torres. (2010). Descubriendo la creatividad en estudiantes universitarios: preferencias $\mathrm{y}$ tendencias mediante la prueba DTC. Revista Iberoamericana de Educación Número 54. Volumen 2, noviembre 2010. https://www.jstor.org/stable/i.ctt1k235pn

GÓMEZ Cumpa José. (2005). Desarrollo de la creatividad. Módulo 4 Maestría en psicopedagogía cognitiva. Universidad Nacional Pedro Ruiz Gallo. 
RECIE. Revista Electrónica Científica de Investigación Educativa Vol. 4, núm. 2, enero-diciembre 2019, pp. 1337-1348.

Fondo Editorial Universitario. Lambayeque www.academia.edu/14539330/Desarrollo de la creatividad

GUILERA, Agüera Llorenc. (2011). Anatomía de la creatividad, FUNDIT Escola Superior de Disseny ESDY de la Universidad Ramón Llull. Talleres Gráficos Vigor S. A. España. 241 pp.

LARRAZ, Rábanos Natalia y Allueva Torres Pedro. (2012). Efectos de un programa para desarrollar las habilidades creativas Electronic Journal of Research in Educational PsyChology. Vol.10, núm. 28. Universidad de Almería. España. 1139 -1158 pp.

MARÍN García Teresa. (2010). Teoría sobre creatividad. 67 pp. www.imaginar.org/taller/th/2 manuales/Teoría creatividad.pdf02/ $\underline{05 / 2016 .}$.

NICKERSON, Raymond et.al. (1998). Enseñar a pensar: Aspectos de la actividad intelectual. Temas de Educación. Paidós. 3ra impresión. España. 398 pp.

ROMERO, Rosario, Cueva, Henry \& Barbosa, Luis, (2014) La gimnasia cerebral como estrategia para el desarrollo de la creatividad en los estudiantes. Omnia, Volumen 2, Número 3, 89 - 91. http://www.redalyc.org/pdf/737/73737091006.pdf

SÁNCHEZ, Dorante Ludivina y Aguilar Castillos Gildardo. (2009). Taller de habilidades de pensamiento crítico y creativo. Universidad Veracruzana

www.uv.mx/personal/gcatana/files/2013/06/antologia-del-cursode-hp.pdf

SÁNCHEZ, Escobedo Pedro Antonio. et.al. (2009). Validez y confiabilidad de un instrumento para medir la creatividad en adolescentes. Revista Iberoamericana de Educación Núm. 50. Octubre. www.redalyc.org/pdf/2831/283121721004.pdf

VELÁZQUEZ, Burgos Bertha Marlen. et.al. (2010). La creatividad como práctica para el desarrollo del cerebro total. Revista Tábula Rasa Num.13. julio-diciembre. Universidad Colegio Mayor de Cundinamarca. Colombia. www.revistatabularasa.org/numero13/13Calle.pdf

El desarrollo de la personalidad creativa en el estudiante de la Licenciatura... 